\title{
Analysis of energy restriction and physical activity on brain function: the role of ketone body and brain-derived neurotrophic factor
}

\author{
Chan Ho Park', Yi-Sub Kwak²,* \\ 'Department of Marine Sports, Pukyong National University, Busan, Korea \\ ${ }^{2}$ Department of Physical Education, Dong-Eui Uinversity, Busan, Korea
}

Brain development is a complex process, and stimuli during this development period may modulate the functional maturation of the brain. It has been shown that environmental stimuli, such as physical activity habits, have a beneficial effect on brain development. Endurance exercise and prolonged fasting state are known to improve brain function including cognition. The exact mechanisms of exercise improving brain function are still unknown. However, it can be considered that energy restriction and stressful challenge induced by long-lasting physical ex-

\section{INTRODUCTION}

Brain development is a complex process, and stimuli during this development period may modulate the functional maturation of the brain and determine its lifelong integrity. Human and animal studies have shown that environmental stimuli, such as physical activity habits, have a beneficial effect on brain development (Gomes da silva and Arida, 2015). Exercise has many functions not only in the developmental period of the brain but also in adulthood.

Exercise is known to have many beneficial effects on brain function and energy metabolism. Endurance exercise and prolonged fasting state are known to improve brain function including cognition. It is well known that exercise training improves hippocampus-dependent cognition, however optimum exercise intensity is still undetermined. Inoue et al. (2015) showed that continuous mild exercise training leads to enhanced hippocampus-related memory both healthy adults and subjects with low physical capacity.

The hippocampal-dependent memory-enhancing effect of exer- ercise might cause direct effect on brain function. Upregulation of brain-derived neurotrophic factor and ketone body caused by exercise might be considered as the mechanism of exercise on brain function. In the present study, we discussed on two main topics: "exercise and BDNF" and "exercise and energy restriction."

Keywords: Physical activity, Energy restriction, Brain-derived neurotrophic factor, Ketone body
*Corresponding author: Yi-Sub Kwak (ib https://orcid.org/0000-0003-4545-7250 Department of Physical Education, College of Arts and Sports Science, Dong-Eui University, 176 Eomgwang-ro, Busanjin-gu, Busan 47340, Korea Tel: +82-51-890-1546, Fax: +82-505-182-6915, E-mail: ysk2003@deu.ac.kr Received: June 28, 2017 / Accepted: August 7, 2017 cise is closely related to exercise-induced increase in hippocampal neurogenesis. Physical exercise most effectively promotes adult hippocampal neurogenesis when exercise is aerobic and lasting (Nokia et al., 2016). Physical exercise is also a strong inducer of reactive oxygen species (ROS), and increased ROS stimulates the regulation of antioxidant enzyme systems in the skeletal and cardiac muscles (Park and Kwak, 2016).

The exact mechanisms of exercise improving brain function are still unknown. However, it can be considered that energy restriction and stressful challenge induced by long-lasting physical exercise might cause direct effect on brain function. The neurotrophin family of signal proteins, including brain-derived neurotrophic factor (BDNF), nerve growth factor, neurotrophin-3 and NT- $4 / 5$ determine the survival and differentiation of neuronal cells during development.

BDNF and energy restriction have a great effect on the effects of exercise. In this study, therefore, we discussed two topics: "exercise and BDNF" and "exercise and energy restriction." 


\section{EXERCISE AND BDNF}

BDNF is a type of neurotrophic factors involved in memory or cognitive development, and its expression is increased by exercise. Improving memory function of exercise is particularly associated with increased neurotrophic factors such as BDNF and insulin-like growth factor-1 (IGF-1) (Tonoli et al., 2015). BDNF is a major regulator of synaptic transmission and plasticity in adult synapses in many areas of the central nervous system. BDNF activates synaptic consolidation through transcription and rapid dendritic trafficking of mRNA encoded by the immediate early gene, Arc (Bramham and Messaoudi, 2005). There was a difference in BDNF expression by exercise intensity and distance. High intensity intermittent exercise increased BDNF levels in the blood of male subjects at 1.25 - or $2.5-\mathrm{km}$ distance, while blood interleukin-6 level was higher in 2.5-km distance (Cabral-Santos et al., 2016).

BDNF is involved in the regulation of adult hippocampal neurogenesis, and tyrosine kinase $\mathrm{B}(\operatorname{TrkB})$ is a $\mathrm{BDNF}$ receptor. $\mathrm{BD}$ NF-TrkB signaling is closely related to progression to neural maturation (Donovan et al., 2008). Regular and prolonged exercise can relieve hippocampal-dependent memory loss caused by chronic stress. Hippocampal adenosine monophosphate-activated protein kinase-dependent BDNF induction is required for exercise-induced protection against chronic stress (Kim and Leem, 2016).

During fasting and intense exercise, brain cell energy substrate utilization moves from glucose to ketone 3-hydroxybutyrate (3OHB). Studies show that $3 \mathrm{OHB}$ can protect neurons against excitotoxicity and oxidative stress. $3 \mathrm{OHB}$ metabolism increases mitochondrial respiration leading to altered expression of BDNF in cultured cortical neurons (Marosi et al., 2016).

\section{EXERCISE AND ENERGY RESTRICTION}

BDNF has been associated with exercise as well as energy restriction. Evolution prefers individuals with excellent cognitive and physical abilities under limited food supply conditions. Mattson (2012) suggested that intermittent energy restriction and prolonged exercise optimize brain function. In the hypothalamus, BDNF inhibits food intake and increases energy consumption. BDNF mediates improvements in cognitive function and neuroprotection caused by exercise and energy restriction through promoting synaptic plasticity and neurogenesis in the hippocampus (Mattson, 2012). Energy restriction also improves cardiovascular stress adaptation by increasing brainstem cholinergic activity (Rothman et al., 2012).
Exercise and energy intake modify the plasticity of neural circuits in a way that affects the health of the brain. By enhancing neurogenesis, synaptic plasticity, and nerve stress robustness, exercise and intermittent energy limitation/fasting can optimize brain function and prevent metabolic and neurodegenerative diseases (van Praag et al., 2014). Caloric restriction improved recognition memory, increased gray matter volume in inferior frontal gyrus and hippocampus, and augmented hippocampal resting-state functional connectivity in the postmenopausal obese women (Prehn et al., 2017).

The ketone body is the most energy efficient fuel and has more ATP per mole of substrate than pyruvic acid and increases the free energy released by ATP hydrolysis. The ketone diet improves rats' physical function and cognitive function, suggesting that energy conservation characteristics can help cure the condition of people with metabolic disorders (Murray et al., 2016). In the type 2 diabetic mice, increased ketone body improved mitochondrial function in the hippocampus, and this process was considered as the adaptive mechanisms to maintain energy during interrupted glucose metabolism (Andersen et al., 2017).

In recent studies, the effects of exercise on mitochondrial function have also become known. Exercise improves mitochondrial energy in skeletal muscle as well as in other tissues. Gusdon et al. (2017) suggested that exercise training in old mice could improve brain mitochondrial function by affecting electron transport chain function and mitochondrial dynamics without increasing mitochondrial biogenesis. Exercise-trained men consuming a protein-pacing diet along with multicomponent exercise training (PRISE) improved muscular power, strength, and flexibility (Ives et al., 2017). These effects of exercise are not likely related to growth hormone or BDNF but possibly related to IGF-1 response (Ives et al., 2017).

\section{CONCLUSIONS}

It is well known that long-term endurance exercise and fasting state improve brain function. Although the precise mechanisms for brain function improvement have not yet been elucidated, ketone body and BDNF have been suggested as the main substances of this action. In future studies, it will be necessary to develop technologies that can clinically use these substances, along with the discovery of other substances that enhance brain function. 


\section{CONFLICT OF INTEREST}

No potential conflict of interest relevant to this article was reported.

\section{ACKNOWLEDGMENTS}

This work was supported by Dong-Eui University Grant (2017 03100001).

\section{REFERENCES}

Andersen JV, Christensen SK, Nissen JD, Waagrpetersen HS. Improved cerebral energetic and ketone body metabolism $\mathrm{db} / \mathrm{db}$ mice. J Cereb Blood Flow Metab 2017;37:1137-1147.

Bramham CR, Messaoudi E. BDNF function in adult synaptic plasticity: the synaptic consolidation hypothesis. Prog Neurobiol 2005;76:99-125.

Cabral-Santos C, Castrillón CI, Miranda RA, Monteiro PA, Inoue DS, Campos EZ, Hofmann P, Lira FS. Inflammatory cytokines and BDNF response to high-intensity intermittent exercise: effect the exercise volume. Front Physiol 2016;7:509.

Donovan MH, Yamaguchi M, Eisch AJ. Dynamic expression of TrkB receptor protein on proliferating and maturing cells in the adult mouse dentate gyrus. Hippocampus 2008;18:435-439.

Gomes da Silva S, Arida RM. Physical activity and brain development. Expert Rev Neurother 2015;15:1041-1051.

Gusdon AM, Callio J, Distefano G, O'Doherty RM, Goodpaster BH, Coen PM, Chy CT. Exercise increases mitochondrial complex I activity and DRP1 expression in the brains of aged mice. Exp Gerontol 2017;18;90: $1-13$.

Inoue K, Hanaoka Y, Nishijima T, Okamoto M, Chang H, Saito T, Soya H. Long-term mild exercise training enhances hippocampus-dependent memory in rats. Int J Sports Med 2015;36:280-285.

Ives SJ, Norton C, Miller V, Minicucci O, Robinson J, O’Brien G, Escudero

D, Paul M, Sheridan C, Curran K, Rose K, Robinson N, He F, Arciero

PJ. Multi-modal exercise training and protein-pacing enhances physical performance adaptations independent of growth hormone and BDNF but may be dependent on IGF-1 in exercise-trained men.
Growth Horm IGF Res 2017;32:60-70.

Kim DM, Leem YH. Chronic stress-induced memory deficits are reversed by regular exercise via AMPK-mediated BDNF induction. Neuroscience 2016;2:324:271-285.

Marosi K, Kim SW, Moehl K, Scheibye-Knudsen M, Cheng A, Cutler R, Camandola S, Mattson MP. 3-Hydroxyrate regulates energy metabolism and induces BDNF expression in cerebral cortical neurons. J Neurochem 2016;139:769-781.

Mattson MP. Energy intake and exercise as determinants of brain health and vulnerability to injury and disease. Cell Metab 2012;16:706-722.

Murray AJ, Knight NS, Cole MA, Cochlin LE, Carter E, Tchabanenko K, Pichulik T, Gulston MK, Atherton HJ, Schroeder MA, Deacon RM, Kashiwaya Y, King MT, Pawlosky R, Rawlins JN, Tyler DJ, Griffin JL, Robertson J, Veech RL, Clarke K. Novel ketone diet enhances physical and cognitive performance. FASEB J 2016;30:4021-4032.

Nokia MS, Lensu S, Ahtiainen JP, Johansson PP, Koch LG, Britton SL, Kainulainen $\mathrm{H}$. Physical exercise increases adult hippocampal neurogenesis in male rats provided it is aerobic and sustained. J Physiol 2016; 594:1855-1873.

Park SY, Kwak YS. Impact of aerobic and anaerobic exercise training on oxidative stress and antioxidant defense in athletes. J Exerc Rehabil 2016;12:113-117.

Prehn K, Jumpertz von Schwartzenberg R, Mai K, Zeitz U, Witte AV, Hampel D, Szela AM, Fabian S, Grittner U, Spranger J, Flöel A. Caloric restriction in older adults-differential effects of weight loss and reduced weight on brain structure and function. Cereb Cortex 2017;27: 1765-1778.

Rothman SM, Griffioen KJ, Wan R, Mattson MP. Brain-derived neurotrophic factor as a regulator of systemic and brain energy metabolism and cardiovascular health. Ann N Y Acad Sci 2012;1264:49-63.

Tonoli C, Heyman E, Buyse L, Roelands B, Piacentini MF, Bailey S, pattyn N, Berthoin S, Meeusen R. Neurotrophins and cognitive functions in T1D compared with healthy controls: effects of a high-intensity exercise. Appl Physiol Nutr Metab 2015;40:20-27.

van Praag H, Fleshner M, Schwartz MW, Mattson MP. Exercise, energy intake, glucose homeostasis, and the brain. J Neurosci 2014;34:1513915149 . 